\title{
Composição florística de duas áreas de Caatinga da Chapada do Araripe
}

\author{
Floristic composition of two areas of Caatinga in Chapada do Araripe \\ Composición florística de áreas de Caatinga en Chapada do Araripe
}

\author{
José Fábio de Oliveira Sousa \\ ORCID: https://orcid.org/0000-0001-6157-6554 \\ Universidade Regional do Cariri, Brasil \\ E-mail: fabio.oliveira@aluno.ufca.edu.br \\ Arycelle Alves de Oliveira \\ ORCID: https://orcid.org/0000-0001-7574-5208 \\ Universidade Regional do Cariri, Brasil \\ E-mail: arycellealves@hotmail.com \\ Natália Barbosa Campos \\ ORCID: https://orcid.org/0000-0003-1805-6216 \\ Universidade Regional do Cariri, Brasil \\ E-mail: campos.b.natalia@gmail.com \\ José Weverton Almeida-Bezerra \\ ORCID: https://orcid.org/0000-0002-0966-9750 \\ Universidade Regional do Cariri, Brasil \\ E-mail: weverton.almeida@urca.br \\ Viviane Bezerra da Silva \\ ORCID: https://orcid.org/0000-0003-0581-2609 \\ Universidade Regional do Cariri, Brasil \\ E-mail: viviane.silva@urca.br \\ Marcio Pereira do Nascimento \\ ORCID: https://orcid.org/0000-0001-5838-4818 \\ Universidade Regional do Cariri, Brasil \\ E-mail: marcio.nascimento@urca.br \\ Priscilla Augusta de Sousa Fernandes \\ ORCID: https://orcid.org/0000-0001-7357-1013 \\ Universidade Regional do Cariri, Brasil \\ E-mail: prisciasf@gmail.com \\ Allyson Francisco dos Santos \\ ORCID: https://orcid.org/0000-0001-8635-6398 \\ Secretaria de Educação e Esportes de Pernambuco, Brasil \\ E-mail: allysons@outlook.com.br \\ Jamile Maria Pereira Bastos Lira de Vasconcelos \\ ORCID: https://orcid.org/0000-0002-0898-0643 \\ Secretaria de Educação e Esportes de Pernambuco, Brasil \\ E-mail: jamilebastoslira@gmail.com \\ Maria Renata Furtado de Sousa \\ ORCID: https://orcid.org/0000-0002-2902-2436 \\ Universidade Regional do Cariri, Brasil \\ E-mail: rfurtado526@gmail.com \\ Maria Arlene Pessoa da Silva \\ ORCID: https://orcid.org/0000-0002-2643-2106 \\ Universidade Regional do Cariri, Brasil \\ E-mail: arlene.pessoa@urca.br \\ Ana Cleide Alcântara Morais Mendonça \\ ORCID: https://orcid.org/0000-0002-0199-177X \\ Universidade Regional do Cariri, Brasil \\ E-mail: anninhamenininha@hotmail.com
}

\section{Resumo}

A Caatinga é a principal formação vegetal do nordeste brasileiro ocupando maior parte da sua área sendo de total importância para a região, possui muitas espécies endêmicas, porém uma pequena porcentagem da sua extensão é protegida por leis ambientais. É nela que se insere a Chapada do Araripe, presente nos estados do Piauí, Pernambuco e Ceará, sendo essa de uma enorme diversidade vegetal, possui diversas fitofisionomias como: Cerrado, Cerradão, Carrasco, Mata Úmida e Caatinga. Objetivou-se com este trabalho, descrever a flora de duas áreas de Caatinga da Chapada do Araripe. As coletas das plantas foram realizadas entre os meses de setembro de 2015 a maio de 2017 . O material foi prensado em campo, desidratado e herborizado. Posteriormente foi encaminhado ao Herbário Caririense Dárdano de Andrade-Lima (HCDAL), da Universidade Regional do Cariri - URCA, para depósito e identificação. 
Foram registradas 126 espécies pertencentes a 78 gêneros e 35 famílias. As famílias mais representativas em números de espécies foram: Fabaceae (23 spp.); Malvaceae (15 spp.) e Euphorbiaceae (10 spp.) e os gêneros mais representativos foram: Sida (10 spp.); Croton (9 spp.); Solanum (5 spp.) e Mimosa (4 spp.). Os resultados obtidos revelam uma grande diversidade para a Chapada do Araripe, comparado com outros estudos realizados na região. Pode-se perceber ainda que Fabaceae e Euphorbiaceae sempre estão em posição de destaque em estudos florísticos desenvolvidos na Caatinga. O presente estudo pode contribuir de forma significativa para futuros projetos de conservação, fornecendo dados sobre a diversidade vegetal do município.

Palavras-chave: Diversidade, Endemismo, Fabaceae.

\begin{abstract}
The Caatinga is the main vegetation formation in northeastern Brazil, occupying most of its area and being of total importance to the region, it has many endemic species, but a small percentage of its extension is protected by environmental laws. It is in it that the Chapada do Araripe is inserted, present in the states of Piauí, Pernambuco and Ceará, which has an enormous plant diversity, has several phytophysiognomies such as: Cerrado, Cerradão, Carrasco, Humid Forest and Caatinga. The objective of this work was to describe the flora of two areas of Caatinga in Chapada do Araripe. Plant collections were carried out between September 2015 and May 2017. The material was pressed in the field, dehydrated and herborized. It was later sent to the Herbarium Caririense Dárdano de Andrade-Lima (HCDAL), of the Regional University of Cariri - URCA, for deposit and identification. 126 species belonging to 78 genera and 35 families were recorded. The most representative families in number of species were: Fabaceae (23 spp.); Malvaceae (15 spp.) and Euphorbiaceae (10 spp.) and the most representative genera were: Sida (10 spp.); Croton (9 spp.); Solanum (5 spp.) and Mimosa (4 spp.). The results obtained reveal a great diversity for the Chapada do Araripe, compared to other studies for the area. It can also be seen that Fabaceae and Euphorbiaceae are always in a prominent position in floristic studies for the Caatinga. The present study can significantly contribute to future conservation projects, providing data on the municipality's plant diversity.
\end{abstract}

Keywords: Diversity, Endemism, Fabaceae.

\title{
Resumen
}

La Caatinga es la principal formación vegetal en el noreste de Brasil, ocupa la mayor parte de su área y siendo de total importancia para la región, tiene muchas especies endémicas, pero un pequeño porcentaje de su extensión está protegido por leyes ambientales. Es en él que se inserta la Chapada do Araripe, presente en los estados de Piauí, Pernambuco y Ceará, que tiene una enorme diversidad vegetal, tiene varias fitofisiognomías como: Cerrado, Cerradão, Carrasco, Bosque Húmedo y Caatinga. El objetivo de este trabajo fue describir la flora de dos áreas de Caatinga en Chapada do Araripe. Las recolecciones de plantas se realizaron entre septiembre de 2015 y mayo de 2017. El material fue prensado en campo, deshidratado y herborizado. Posteriormente fue enviado al Herbario Caririense Dárdano de Andrade-Lima (HCDAL), de la Universidad Regional de Cariri - URCA, para su depósito e identificación. Se registraron 126 especies pertenecientes a 78 géneros y 35 familias. Las familias más representativas en número de especies fueron: Fabaceae (23 spp.); Malvaceae (15 spp.) y Euphorbiaceae (10 spp.) y los géneros más representativos fueron: Sida (10 spp.); Croton (9 spp.); Solanum (5 spp.) y Mimosa (4 spp.). Los resultados obtenidos revelan una gran diversidad para la Chapada do Araripe, en comparación con otros estudios para el área. También se puede ver que las Fabaceae y Euphorbiaceae están siempre en una posición destacada en los estudios florísticos de la Caatinga. El presente estudio puede contribuir significativamente a futuros proyectos de conservación, proporcionando datos sobre la diversidad vegetal en el municipio.

Palabras clave: Diversidad, Endemismo, Fabaceae.

\section{Introdução}

A falta de conhecimento e o uso inadequado dos recursos provenientes da Caatinga, uma floresta tropical sazonalmente seca, tem apresentado várias consequências, como a perca da biodiversidade, de modo a levar a extinção de espécies. A Caatinga, representa o quarto domínio fitogeográfico de maior extensão territorial do Brasil, chegando a aproximadamente $850.000 \mathrm{Km}^{2}$, sendo exclusivamente brasileiro apresenta grande faixa de endemismo, porém menos de $2 \%$ da sua cobertura vegetal é protegida por unidades de conservação de proteção integral (Siqueira-Filho et al., 2009). Estando presente nos estados da Paraíba, Ceará, Pernambuco, Rio Grande do Norte, Sergipe, Piauí, Alagoas, Bahia e Minas Gerais e abrangendo cerca de $54 \%$ da região Nordeste e $11 \%$ do território brasileiro (Brasil, 2005), esse domínio fitogeográfico é bastante importante, pois apresenta um alto padrão de biodiversidade e um grande número de espécies endêmicas (Ferraz et al., 2013).

As plantas dessa floresta desenvolveram ao longo da sua história evolutiva caracteres morfológicos, anatômicos e 
fisiológicos, especializados em garantir sua sobrevivência sob condições adversas nesse ambiente, principalmente o estresse hídrico (Silva et al., 2010). Queiroz (2009) a descreve como uma formação onde as espécies possuem baixo porte, com folhas decíduas e as árvores bem ramificadas, apresentando espinhos ou acúleos.

É nesse domínio que se insere a Chapada do Araripe, abrangendo partes dos estados do Piauí, Pernambuco e Ceará, detendo sua maior extensão no último (Fernandes \& Bezerra, 1990). Sua constituição vegetal diversificada, revela diversas fitofisionomias como: Cerrado, Cerradão, Carrasco, Mata Úmida e Caatinga, com o Cerrado sendo a vegetação dominante (Sampaio, Andrade-Lima \& Gomes 1981; Radambrasil, 1981). Há décadas a vegetação da Chapada do Araripe vem sofrendo exploração antrópica o que reflete a enorme perda da composição genética, muitas das quais pouco se tem conhecimento (Alencar et al., 2012).

A Chapada do Araripe, tem uma superfície estrutural tabular, com seu platô no nível de 800 a $1000 \mathrm{~m}$ de altura e possui em seu subsolo um grande aquífero (Alencar, Silva \& Barros; 2007). Sua extensão varia cerca de 35 a 60 km de norte a sul e cerca de $180 \mathrm{~km}$ de leste a oeste com uma inclinação de aproximadamente $300 \mathrm{~m}$ na sua superfície do sentido oriental para o ocidental, sua altitude média varia de 690 à 1005 m (Santos et al., 2009). Detém uma enorme beleza mostrada pelas suas formas geológicas, suas nascentes de água doce, sua diversa vegetação e seu clima semiárido, que se apresenta contrastando com o verde das matas e suas águas (Viana \& Neumann; 2006). Por outro lado, o clima também tem forte influência sobre a cobertura vegetal da Chapada do Araripe, diferenciando-se de forma significativa nos setores oriental e ocidental. O clima é tropical chuvoso no setor oriental e quente e semiárido no ocidental (Mendonça et al., 2000).

Desde o século XIX, a vegetação da Chapada do Araripe vem sendo explorada pelo ser humano, naturalistas já tinham grande interesse pela riqueza vegetal da região. Na época já relatava-se o uso da madeira de Caryocar coriaceum Witmm (pequi) para a produção de moendas, as sementes Magonia pubescens A. St. Hil (tingui) para a produção de sabão, da madeira de Cedrela fissilis Vell (cedro) para a produção de móveis e dos frutos de Psidium bergianum (O.Berg) Nied (araçá), para a alimentação, além da utilização de algumas espécies na medicina popular cultural de seus habitantes e para ornamentações (Gardner, 1849).

A área de Caatinga localizada na Chapada do Araripe detém uma grande diversidade em espécies vegetais, porém, nos últimos anos vem se tendo uma redução muito acelerada da sua constituição vegetal e estudos que descrevam sua flora ainda são escassos. Sendo que no estado do Pernambuco, a Chapada vem sendo alvo de desmatamento devido a grande quantidade de loteamentos de terras que estão sendo feitos, extração de madeira e desmatamentos com fins agropecuários o que está resultando na perda dos seus recursos naturais e uma grande diminuição na sua cobertura vegetal. Portanto, objetivou-se com este trabalho, descrever a flora de duas áreas de Caatinga da Chapada do Araripe, localizadas em dois Sítios vizinhos no município de Moreilândia, estado de Pernambuco.

\section{Metodologia}

\section{1 Área de estudo}

O levantamento florístico foi realizado em duas localidades: Área 1 - Sítio Mata Nova (07037'51"S e 39³3'04" W) e

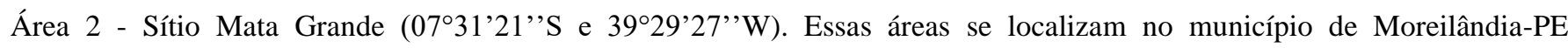
(07³7'51"S e 39³3'04" W). Situado na mesorregião do Sertão Pernambucano o município apresenta área de 404,57 Km² e população de 11.242 habitantes (IBGE, 2016).

O clima semiárido provoca uma temperatura média anual de $24,7^{\circ} \mathrm{C}$. A sua altitude é de $502 \mathrm{~m}$. Compreendendo o bioma Caatinga, sua vegetação é exclusivamente de Floresta Caducifólia e Caatinga Hipoxerófila. Sua geologia é formada por maciços imponentes caracterizando um relevo pouco acidentado e seus solos são de alta fertilidade. A parte norte está inserida na unidade geoambiental das Chapadas Altas (IBGE, 2016). 


\subsection{Coleta do material}

As coletas do material botânico foram realizadas mensalmente entre os meses de setembro de 2015 a maio de 2017. Os espécimes vegetais foram coletados em estágio reprodutivo, sendo coletados cinco ramos férteis de cada espécie e utilizouse aparelho de GPS para georreferenciar os pontos de coleta. O material foi prensado em campo, desidratado e posteriormente foi encaminhado ao Herbário Caririense Dárdano de Andrade-Lima (HCDAL) da Universidade Regional do Cariri - URCA, onde foi herborizado, tombado e identificado por especialistas, literatura especializada, chaves de identificação, e por comparação com exemplares do herbário supracitado.

\section{Resultados e Discussão}

Foram registradas 35 famílias, 78 gêneros e 126 espécies, dos mais variados hábitos: ervas, subarbustos, arbustos, trepadeiras e árvores. Tabela 1. 


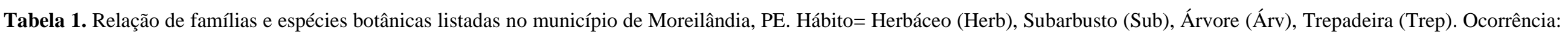

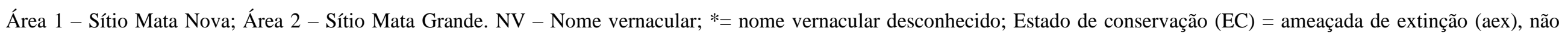
avaliada (nav), pouco preocupante (ppr), quase ameaçada (qam), **=táxon apena a nível de gênero, ***= táxon apenas a nível de família.

\begin{tabular}{|c|c|c|c|c|c|c|c|}
\hline ESPÉCIE & FAMÍLIA & HÁBITO & $\mathbf{N V}$ & $\begin{array}{l}\text { OCORF } \\
\text { Área } 1\end{array}$ & $\begin{array}{r}\text { ÊNCIA } \\
\text { Área } 2\end{array}$ & EC & $\begin{array}{c}\mathbf{N}^{\circ} \mathrm{DE} \\
\text { TOMBO }\end{array}$ \\
\hline Ruellia panicula & Acanthaceae & Sub & $*$ & $\mathrm{X}$ & $\mathrm{X}$ & nav & 12.481 \\
\hline Alternanthera brasiliana (L.) Kuntze & Amaranthaceae & Sub & Quebrapanela & $\mathrm{X}$ & $\mathrm{X}$ & nav & 12.471 \\
\hline Amaranthu ssp. & Amaranthaceae & Herb & Brêdo & $\mathrm{X}$ & $\mathrm{X}$ & $* *$ & 12.488 \\
\hline Rhodophiala cf. laeta Phil & Amaryllidaceae & Herb & Cebola-brava & $\mathrm{X}$ & $\mathrm{X}$ & nav & 12.420 \\
\hline Myracrodruon urundeuva Allemão & Anacardiaceae & Árv & Aroeira & - & $\mathrm{X}$ & aex & 12.582 \\
\hline Annona coriácea Mart. & Annonaceae & Árv & Araticum & $\mathrm{X}$ & $\mathrm{X}$ & nav & 12.447 \\
\hline Annona leptopetala (R.E.Fr.) H. Rainer & Annonaceae & Árv & Pinha-do-mato & $\mathrm{X}$ & $\mathrm{X}$ & nav & 12.460 \\
\hline Ditassacapillaris E. Fourn. & Apocynaceae & Trep & $*$ & $\mathrm{X}$ & - & nav & 12.404 \\
\hline Ditassaglazioui E. Fourn. & Apocynaceae & Trep & $*$ & $\mathrm{X}$ & - & nav & 12.402 \\
\hline Himathanthus drasticus (Mart.) Plumel & Apocynaceae & Árv & Janaguba & - & $\mathrm{X}$ & nav & 12.300 \\
\hline Secondatia floribunda DC. & Apocynaceae & Trep & Catuaba-de-cipó & $\mathrm{X}$ & - & nav & 12.419 \\
\hline Acanthospermu maustrale (Loefl.) Kuntze & Asteraceae & Herb & Carrapicho & - & $\mathrm{X}$ & nav & 12.854 \\
\hline Acanthospermum hispidum DC. & Asteraceae & Herb & $\begin{array}{l}\text { Carrapicho-de- } \\
\text { cangalha }\end{array}$ & $\mathrm{X}$ & $\mathrm{X}$ & nav & 12.778 \\
\hline Aspilia sp. & Asteraceae & Sub & Mal-me-quer & - & $\mathrm{X}$ & $* *$ & 12.399 \\
\hline Bidens pilosa $\mathrm{L}$ & Asteraceae & Herb & Picão & $\mathrm{X}$ & - & nav & 12.510 \\
\hline Barroso abetonicae formis (DC.) R. M. King. \& H. Rob & Asteraceae & Sub & Mentraste & $\mathrm{X}$ & $\mathrm{X}$ & nav & 12.370 \\
\hline Asteraceae 1 & Asteraceae & Sub & Balái-de-velho & $\mathrm{X}$ & $\mathrm{X}$ & $* * *$ & 12.907 \\
\hline Asteraceae 2 & Asteraceae & Sub & $*$ & - & $\mathrm{X}$ & $* * *$ & 12.913 \\
\hline Anemopaegma leave DC. & Bignoniaceae & Trep & Manacá & $X$ & $\mathrm{X}$ & nav & 12.299 \\
\hline Fridericia chica (Bonpl.) L.G. Lohmann & Bignoniaceae & Trep & $\begin{array}{l}\text { Cipó-de-arco-de- } \\
\text { forno }\end{array}$ & $\mathrm{X}$ & $\mathrm{X}$ & nav & 12.417 \\
\hline Jacaranda jasminoides (Thunb.) Sandwith & Bignoniaceae & Arb & Jacarandá & - & $\mathrm{X}$ & nav & 12.380 \\
\hline Bignoniaceae 1 & Bignoniaceae & Arb & $*$ & - & $\mathrm{X}$ & $* * *$ & 12.634 \\
\hline Cordia rufescens DC. & Boraginaceae & Arb & Grão-de-galo & $\mathrm{X}$ & $\mathrm{X}$ & nav & 12.372 \\
\hline Myriopus salzmannii (DC.) Diane \&Hilger & Boraginaceae & Trep & Guerra & $\mathrm{X}$ & $\mathrm{X}$ & nav & 12.392 \\
\hline Varroni acurassavica Jacq. & Boraginaceae & Arb & Erva-baleeira & - & $\mathrm{X}$ & nav & 12.719 \\
\hline Varronia globosaJacq. & Boraginaceae & Arb & Moleque-duro & $\mathrm{X}$ & $\mathrm{X}$ & nav & 12.453 \\
\hline Harrisia adscendens (Gürke) Britton \& Rose & Cactaceae & Arb & Rabo-de-raposa & $\mathrm{X}$ & $\mathrm{X}$ & $\mathrm{ppr}$ & 12.382 \\
\hline Evolvulus glomeratus Nees\& Mart. & Convolvulaceae & Herb & $* 1$ & $\mathrm{X}$ & $\mathrm{X}$ & nav & 12.909 \\
\hline Ipomoea blanchetii Choisy & Convolvulaceae & Trep & Cipó-de-caçuá & $X$ & $X$ & nav & 12.503 \\
\hline
\end{tabular}


Erythroxylum barbatum O. E. Schulz

Croton adamantinus Müll. Arg.

$$
\text { Croton glandulosus L. }
$$

Croton heliotropiifolius Kunth

Croton jacobinensis Baill.

Croton limae A.P. Gomes, H-F Sales, P.E. Berry

$$
\begin{aligned}
& \text { Croton sp. } 1 \\
& \text { Croton sp. } 2 \\
& \text { Croton sp. } 3 \\
& \text { Croton sp. } 4 \\
& \text { Sebastiania sp. }
\end{aligned}
$$

Bauhinia cheilantha (Bong.) Steud.

Bowdichia virgilioides Kunth

Cassia ferrugínea (Schrad.) Schrad. ex DC.

Chamaecrista rotundifolia (Pers.) Greene

Copaifera langsdorffii Desf.

Dahlstedtia araripensis (Benth.) M.J. Silva \& A.M.G.

$$
\text { Azevedo }
$$

Dimorphandra gardneriana Tul.

Dioclea grandiflora Mart. ex Benth.

$$
\text { Dioclea } \mathrm{sp} \text {. }
$$

Hymenaea courbaril L.

Mimosa sensitiva $\mathrm{L}$.

Mimosa somnians Humb. \& Bonpl. Ex Willd.

Mimosa verrucosa Benth.

$$
\text { Mimosa sp. }
$$

Plathymenia reticulata Benth.

Parkia platycephalla Benth.

Senegalia polyphylla (DC.) Britton \& Rose

$$
\text { Senegalia sp. }
$$

Senna cearenses Afr. Fern.

Senna macranthera (DC. ex Collad.) H.S. Irwin

$$
\text { \&Barneby }
$$

Swartzia flaemingii Raddi

$$
\text { Zornia sp. }
$$

Fabaceae 1

Medusantha martiusii (Benth.) Honley\& J.F.B. Pastore

$\begin{array}{lc}\text { Erythroxylaceae } & \text { Arb } \\ \text { Euphorbiaceae } & \text { Arb } \\ \text { Euphorbiaceae } & \text { Herb } \\ \text { Euphorbiaceae } & \text { Arb } \\ \text { Euphorbiaceae } & \text { Arb } \\ \text { Euphorbiaceae } & \text { Arb } \\ \text { Euphorbiaceae } & \text { Arb } \\ \text { Euphorbiaceae } & \text { Arb } \\ \text { Euphorbiaceae } & \text { Arb } \\ \text { Euphorbiaceae } & \text { Arb } \\ \text { Euphorbiaceae } & \text { Arb } \\ \text { Fabaceae } & \text { Arb } \\ \text { Fabaceae } & \text { Arv } \\ \text { Fabaceae } & \text { Arv } \\ \text { Fabaceae } & \text { Herb } \\ & \\ \text { Fabaceae } & \text { Arv } \\ \text { Fabaceae } & \text { Arv } \\ & \\ \text { Fabaceae } & \text { Arv } \\ \text { Fabaceae } & \text { Trep } \\ \text { Fabaceae } & \text { Trep } \\ \text { Fabaceae } & \text { Arv } \\ \text { Fabaceae } & \text { Herb } \\ \text { Fabaceae } & \text { Arv } \\ \text { Fabaceae } & \text { Arv } \\ \text { Fabaceae } & \text { Herb } \\ \text { Fabaceae } & \text { Arv } \\ \text { Fabaceae } & \text { Arb } \\ \text { Fabaceae } & \text { Arb } \\ \text { Fabaceae } & \text { Arv } \\ \text { Fabaceae } & \text { Arb } \\ & \\ \text { Fabaceae } & \text { Arb } \\ \text { Fabaceae } & \\ \text { Fabaceae } & \text { Fabaceae } \\ \text { Lamiaceae } & \text { Arb } \\ \text { Fabae } & \text { Arb }\end{array}$

Arb

Arb

Arb

Arb

Arb

Arb

Arb

Arb

Arb

Arv

Arv

Arv

Arv

Arv

Trep

Arv

Herb

Arv

Arv

Arv

Arb

Arb

Arb

Arb

Arb

Batinga
$*$
Marmeleiro
Velame
Marmeleiro
Carrasco-preto
Velame
Canelinha
Velame
Velame-preto

Pata-de-vaca

Sucupira

Canafista

Amendoin-decarcará

Pau-d'óleo

Angelim

Faveira

Mucunã

Jatobá

Malicia

Jiquirí

Abre-e-fecha

Amarelo

Visgueiro

Cramunzé

Arapiraca

Canafístula-de-

bezouro

Canafístula-de-boi

\section{Banheira}

$$
\text { * }
$$

Cidreira-brava
12.424

2.707

12.806

12.395

12.400

12.490

12.811

12.446

12.396

12.403

12.450

12.434

12.638

12.908

12.375

12.637

12.305

12.506

12.332

12.283

12.736

12.485

12.474

2.812

12.805

12.397

12.546

12.716

12.649

12.489

12.371

12.390

12.302

12.499 


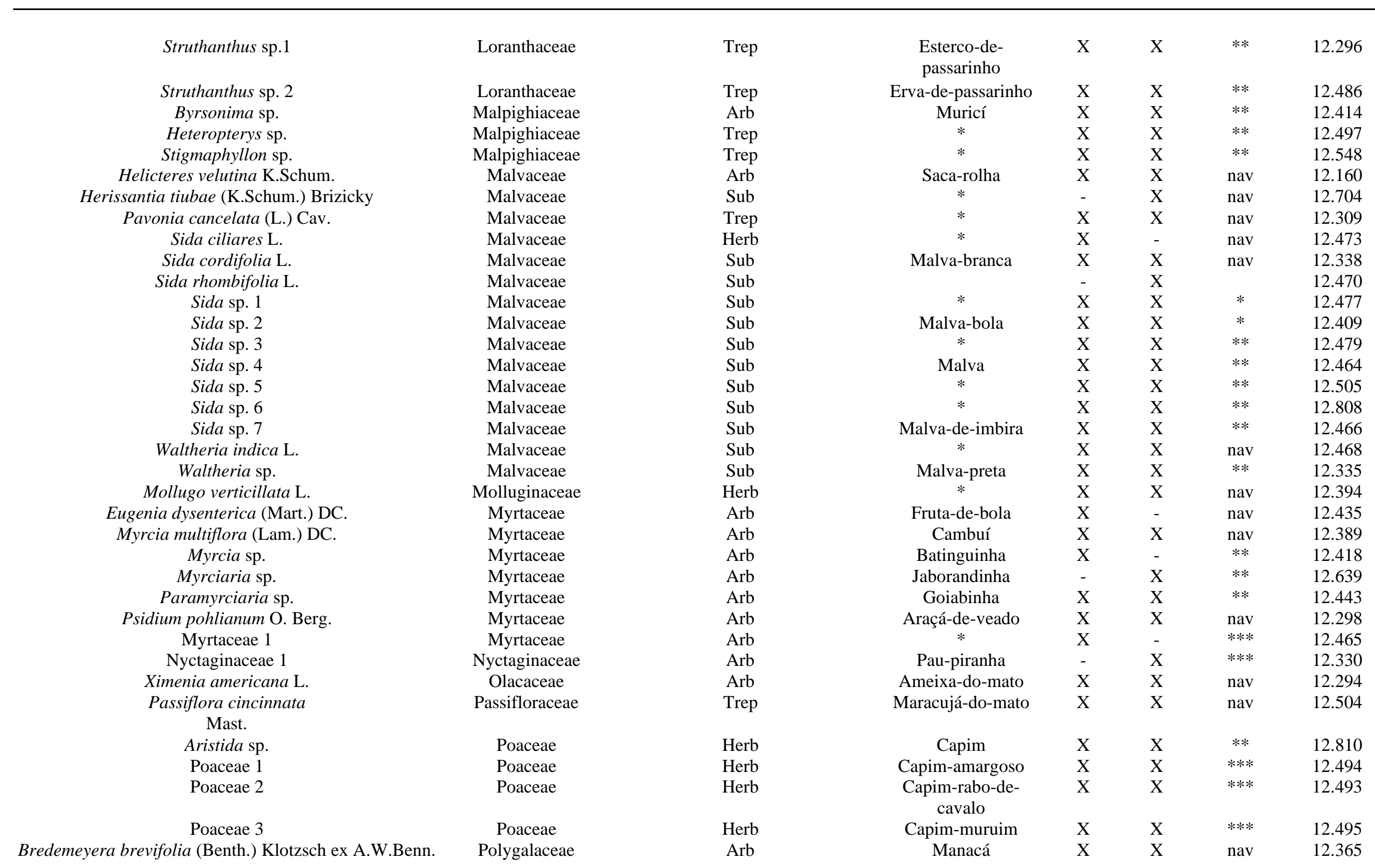


Research, Society and Development, v. 10, n. 13 e506101321398, 2021

(CC BY 4.0) | ISSN 2525-3409 | DOI: http://dx.doi.org/10.33448/rsd-v10i13.21398

\begin{tabular}{|c|c|c|c|c|c|c|c|}
\hline Colubrina cordifolia Reissek & Rhamnaceae & Arb & Saboeiro & $\mathrm{X}$ & $\mathrm{X}$ & nav & 12.549 \\
\hline Borreria verticillata (L.) G.Mey. & Rubiaceae & Herb & Corredeira & $X$ & $\mathrm{X}$ & nav & 12.738 \\
\hline Borreria virgata Cham. \& Schltdl. & Rubiaceae & Herb & Corredeira & $\mathrm{X}$ & $\mathrm{X}$ & nav & 12.487 \\
\hline $\begin{array}{l}\text { Coussarea hydrangeifolia } \\
\text { (Benth.) Müll. Arg. }\end{array}$ & Rubiaceae & Arb & Angélica & $\mathrm{X}$ & - & nav & 12.498 \\
\hline Hexasepalum teres (Walter) J.H. Kirkbr. & Rubiaceae & Herb & $*$ & $\mathrm{X}$ & $\mathrm{X}$ & nav & 12.452 \\
\hline $\begin{array}{c}\text { Margaritopsis carrascoana (Delpetre \& E. B. Souza) } \\
\text { C.M.Taylor \& E.B.Souza }\end{array}$ & Rubiaceae & Arb & $\begin{array}{l}\text { Alecrim-do- } \\
\text { carrasco }\end{array}$ & & & nav & 12.712 \\
\hline Zanthoxylum gardneri Engl. & Rutaceae & Arb & Laranjinha & $X$ & $\mathrm{X}$ & nav & 12.312 \\
\hline Casearia commersoniana Cambess. & Salicaceae & Arb & $\begin{array}{l}\text { Canelinha-de- } \\
\text { nambú }\end{array}$ & $X$ & $\mathrm{X}$ & nav & 12.433 \\
\hline Casearia sylvestris $\mathrm{Sw}$. & Salicaceae & Arb & Guaçatonga & $X$ & $\mathrm{X}$ & nav & 12.405 \\
\hline Magonia pubescens A. St.-Hil. & Sapindaceae & Arv & Tingui & $\mathrm{X}$ & - & nav & 12.367 \\
\hline Matayba guianensis Aubl. & Sapindaceae & Arb & Pitomba-brava & $X$ & $\mathrm{X}$ & nav & 12.339 \\
\hline Serjania lethalis A. St.-Hil. & Sapindaceae & Trep & Cipó-uva & $X$ & $\mathrm{X}$ & nav & 12.291 \\
\hline Talisia esculenta (Cambess.) Radlk. & Sapindaceae & Arv & Pitomba & $X$ & $\mathrm{X}$ & nav & 12.653 \\
\hline Chrysophyllum arenarium Allemão & Sapotaceae & Arv & Cajazinha & $X$ & $X$ & qam & 12.492 \\
\hline Scrophulariaceae 1 & Scrophulariaceae & Herb & $*$ & $\mathrm{X}$ & - & $* * *$ & 12.368 \\
\hline Cestrum $\mathrm{sp}$ & Solanaceae & Arb & $*$ & $\mathrm{X}$ & $\mathrm{X}$ & $* *$ & 12.387 \\
\hline Solanum paludosum Moric. & Solanaceae & Arb & Jurubeba-branca & $X$ & $\mathrm{X}$ & nav & 12.776 \\
\hline Solanum paniculatum $\mathrm{L}$. & Solanaceae & Arb & $\begin{array}{l}\text { Jurubeba-de- } \\
\text { espinho }\end{array}$ & $\mathrm{X}$ & $\mathrm{X}$ & nav & 12.814 \\
\hline Solanum stipulaceum Willd. Ex Roem. \& Schult. & Solanaceae & Arb & Jurubeba & $\mathrm{X}$ & $\mathrm{X}$ & nav & 12.308 \\
\hline Solanum sp. & Solanaceae & Arb & Jurubeba & $\mathrm{X}$ & $\mathrm{X}$ & $* *$ & 12.385 \\
\hline Piriqueta sidifolia (Cambess.) Urb. & Turneraceae & Arb & & $\mathrm{X}$ & $\mathrm{X}$ & nav & 12.456 \\
\hline Turnera cearenses Urb. & Turneraceae & Arb & & $\mathrm{X}$ & $\mathrm{X}$ & nav & 12.709 \\
\hline Turnera sp. & Turneraceae & Arb & $*$ & $\mathrm{X}$ & $\mathrm{X}$ & $* *$ & 12.337 \\
\hline Lantana camara L. & Verbenaceae & Arb & Camará & $\mathrm{X}$ & $X$ & nav & 12.511 \\
\hline Lantana fucata Lindl. & Verbenaceae & Arb & Camará & $X$ & $X$ & nav & 12.336 \\
\hline
\end{tabular}

Fonte: Autores. 
As famílias mais representativas em número de espécies foram: Fabaceae (23 spp.); Malvaceae (15 spp.); Euphorbiaceae (10 spp.); Asteraceae (7 spp.); Myrtaceae (7 spp.); Solanaceae (6 spp.); Rubiaceae (5 spp.); Apocynaceae (4 spp.); Bignoniaceae (4 spp.); Boraginaceae (4 spp.); Poaceae (4 spp.) e Sapindaceae (4 spp.), as demais famílias apresentaram um número igual ou inferior a três espécies. Em relação os maiores gêneros obtiveram-se: Sida L. (10 spp.); Croton L. (9 spp.); Solanum L. (5 spp.) e Mimosa L. (4 spp.). Os resultados obtidos com o levantamento apresentaram uma grande diversidade presente na Caatinga, revelando um número significativo de espécies na área em questão.

Os dados quanto as famílias mais ricas em espécies nesse estudo corroboram com o levantamento florístico de Pereira-Júnior et al., (2013) em um fragmento de Caatinga em Monteiro, Paraíba, o de Tavares et al. (2016) em áreas com diferentes intensidades de pastagem em Pernambuco, e o de Santos et al. (2017) em um levantamento florísticofitossociológico em área de manejo florestal no município de Desterro, Paraíba; ressalvando a biodiversidade do domínio.

Verificando a flora de um trecho de Cerradão da Chapada do Araripe Alencar et al., (2007) também relataram a família Fabaceae como a mais rica em termos de números de espécies com (17 spp.) e Costa et al., (2004) também relataram a família Fabaceae como mais representativa com (17 spp.) em um levantamento de encrave de Cerrado da Chapada do Araripe. Em diversos levantamentos florísticos em áreas de Caatinga também pôde-se observar que a família Fabaceae apresentou-se de forma bastante notável observado por Pereira Júnior; Andrade; Araújo (2013) em um fragmento de Caatinga em Monteiro, Paraíba; Leitão et al. (2014) em um ambiente transicional Caatinga-Mata Atlântica em região litorânea no Rio Grande do Norte; Lima; Barbosa (2014) na RPPN Fazenda Almas no Cariri paraibano, Paraíba e Calixto Júnior; Drumond (2014) em dois fragmentos de Caatinga na Embrapa Semiárido, Petrolina, Pernambuco. Isso é devido à família apresentar características morfológicas que as auxiliem nessas florestas tropicais secas, tal como o limbo da folha ser dividido em folíolos, de modo que diminui a área de superfície foliar e consequentemente diminui a quantidade de água perdida para o ambiente por meio da evapotranspiração.

Um dado interessante foi o grande número de espécies da Malvaceae ( $8 \mathrm{sp}$. ), comparado com outros levantamentos da Chapada do Araripe, Alencar et al., 2012, verificando a composição florística em um fragmento de floresta (Barbalha - Crato, Ceará), registrou apenas uma espécie, já (Ribeiro-Silva et al., 2012) realizando um levantamento de angiospermas da Floresta Nacional do Araripe, também localizada na Chapada do Araripe, registrou três espécies. Herissantia tiubae (K.Schum.) Brizicky (Malvaceae), foi verificada como uma nova ocorrência para a Chapada do Araripe, ainda não tendo sido registrada em levantamentos da flora na região, onde foi registrado apenas um indivíduo da espécie.

Verificou-se que a espécie Dahlstedtia araripensis (Benth.) M.J. Silva \& A.M.G. Azevedo (Fabaceae) apresentou-se fortemente adaptada nas duas áreas de coleta apresentando um grande número de indivíduos distribuídos territorialmente, Fernandes et al., (1964) relata sua ocorrência restrita ao nordeste brasileiro ocorrendo em formações vegetais como Cerrado, Carrasco e ambientes de altitude como serras e chapadas. Por outro lado, a espécie Cordia rufescensA.DC. (Boraginaceae) foi observada em período reprodutivo durante todos os meses de coleta.

Margaritopsis carrascoana (Delprete\& E. B. Souza) C. M. Taylor \& E. B. Souza é uma espécie arbustiva da família Rubiaceae e endêmica do Nordeste brasileiro ocorrendo nos platôs do Planalto de Ibiapaba e Chapada do Araripe, Ceará (Nascimento et al., 2015), no presente estudo M. carrascoana foi registrada nas duas áreas do estudo onde os indivíduos foram encontrados apenas em locais de vegetação fechada com a presença de árvores altas, locais semelhantes aos que ocorrem mais frequentemente.

O gênero mais representativo na área foi Sida L. (Malvaceae) com 9 spp. O gênero apresenta distribuição cosmopolita e pantropical, onde seus locais de maior ocorrência são os continentes americano e africano onde atualmente apresenta cerca de 200 espécies (Neto, 2014), e no Brasil cerca de 90 espécies distribuídas territorialmente (Bovini, 2013).

No trabalho de Alves et al. (2011) sobre a família Malvaceae no Agreste Paraibano pôde-se observar que o gênero 
Sida L. foi o mais representativo, com 5 spp. e o levantamento da família por Bovini; Carvalho-Okano; Vieira 2001, no Parque Estadual do Rio Doce, Minas Gerais o gênero apresentou 7 spp. sendo o mais representativo.

O gênero Croton L. (Euphorbiaceae) apresenta aproximadamente 1.200 espécies (Govaerts, Frodin \& RadcliffeSmith, 2000), está amplamente distribuído nas regiões tropicais do globo e algumas espécies nativas nas regiões subtropicais e temperadas do norte geográfico. No Brasil possui cerca de 300 dentre elas ervas, arbustos e árvores, são encontradas em formações vegetais abertas, Caatinga e algumas espécies podem ser encontradas em Mata Úmida (Burger \& Huft, 1995; Berry et al., 2005). O gênero além de apresentar o maior número de espécies, aparece mais bem distribuído na área, significando que ele possui uma maior adaptação, e este resultado corrobora com Sanquetta et al., (2014), que analisando a diversidade e estrutura fitossociológica de Caatinga em Brumado - BA, também observou que a maioria dos indivíduos pertenciam ao gênero. Rodal et al., (2008), ressalta que Euphorbiaceae é uma das principais famílias da Caatinga, sendo elas das mais ricas em gêneros, espécies e abundância de plantas.

O gênero Solanum L. é o mais diversificado da família Solanaceae e entre as angiospermas é um dos mais numerosos (Hunziker, 2001). Possui cerca de 1.500 espécies e sua distribuição no globo ocorre na América do Sul, América Central, Austrália e África, porém sua maior faixa endêmica e diversidade está na América do Sul (Nee, 1999; Knapp, 2008). No Brasil apresenta 250 espécies onde cerca de 100 são endêmicas, com distribuição em áreas de Caatinga, Floresta Atlântica e regiões áridas (Agra, 2009).

O gênero Mimosa L. apresenta cerca de 540 espécies distribui-se majoritariamente em região Neotropical com aproximadamente 490 espécies endêmicas e 40 nativas do Velho Mundo (SIMON et al., 2011). Suas espécies são encontradas em vários ambientes, Savanas, Campos, Caatinga e até regiões desérticas do México (Barneby, 1991). A maior diversidade do gênero Mimosa L. concentra-se na América do Sul em países como Brasil, Argentina, Paraguai e Uruguai. No Brasil ocorrem cerca de 320 espécies (Dutra \& Amorim, 2010) e destas 38 estão presentes na Caatinga (Queiroz, 2009).

O presente trabalho também corroborou com Moro et al. (2014) que cita as famílias Fabaceae, Euphorbiaceae e Malvaceae como mais representativas para o domínio Caatinga ao mesmo tempo que cita Croton L. e Mimosa L. como os gêneros mais ricos para o domínio. Croton L. é o maior gênero da Caatinga com 68 spp. (Carneiro-Torres, 2009), precedido de Mimosa L. com 61 spp. (Giulietti et al., 2002).

\section{Conclusão}

Os resultados obtidos na área do estudo revelam uma grande diversidade de espécies para a Chapada do Araripe. O presente estudo pode contribuir de forma significativa para futuros projetos de conservação, fornecendo dados sobre a diversidade de plantas.

\section{Referências}

Agra, M. F. (2009). Solanaceae. In: Alves, M., Araújo, M. F., Maciel, J. R., \& Martins, S. (Orgs.). Flora de Mirandiba. Recife: Associação Plantas do Nordeste, p. 339 - 344, 2009.

Alencar, A. L., Silva, M. A. P., \& Barros, L. M. (2007). Florística e Fitossociologia de uma Área de Cerradão na Chapada do Araripe - Crato - CE. Revista Brasileira de Biociências, 5, 18- 20, 2007.

Alencar, S. R., Silva, M. A. P., Macêdo, D. G., \& Oliveira, A. S. (2012). Composição Florística do Estrato Arbóreo de um Fragmento Florestal da Chapada do Araripe: Subsídio Para Construção de um Banco de Germoplasma. Caderno de Cultura e Ciência, 11(1), 21-24.

Alves, I. M., Dantas, I. C., Melo, J. I. M., \& Felismino, D. C. A. (2011). Família Malvaceae sensu lato em uma Área do Agreste Paraibano, Nordeste do Brasil. BIOFAR (Revista de Biologia e Farmácia), 6(1), 1-20.

Barneby, C. R. (1991). Sensitivae Censitae. A description of the genus Mimosa L. (Mimosaceae) in the New World. Mem. New York Botanic Gardens, 65, 1 -835 . 
Berry, P. E., Hipp, A. L., Wurdack, K. J., Vanee, B., \& Riina, R. (2005). Molecular Phylogenetics of the giant genus Croton and tribe Crotoneae (Euphorbiaceaesensustricto) using ITS and trnL-trnF DNA sequence data. American Journal of Botanic, 92(9), 1520 - 1534.

Bovini, M. G. (2013). Sida. Lista de Espécies da Flora do Brasil. Jardim Botânico do Rio de Janeiro. http://floradobrasil.jbrj.gov.br /jabot/floradobrasil/FB9203.

Bovini, M. G., Carvalho-Okano, R. M., \& Vieira, M. F. (2001). Malvaceae A. Juss. No Parque Estadual do Rio Doce, Minas Gerais, Brasil. Rodriguésia, 52(81), 17-47.

Brasil. (2005). Ministério da Integração Nacional. Nova delimitação do semi-árido brasileiro.

Burger, W., \& Huft, M. (1995). Euphorbiaceae. In: Flora Costaricensi. Fieldiana, 2(36), 1-169.

Calixto Júnior, J. T., \& Drumond, M. A. (2014). Estudo comparativo da estrutura fitossociológica de dois fragmentos de Caatinga em níveis diferentes de conservação. Pesquisa Florestal Brasileira, 34(80), 345-355.

Carneiro-Torres, D. S. (2009). Diversidade de Croton L. (Euphorbiaceae) no Bioma Caatinga (Tese de Doutorado). Programa de Pós-Graduação em Botânica da Universidade Estadual de Feira de Santana, Feira de Santana, BA, Brasil.

Costa, I. R., Araújo, F. S., \& Lima-Verde, L. W. (2004). Flora e aspectos auto ecológicos de um encrave de cerrado na Chapada do Araripe, Nordeste do Brasil. Acta Botânica Brasilica, 18(4), 759-770.

Dutra, V. F., \& Morim, M. P. (2010). Mimosa. Lista de Espécies da Flora do Brasil. Jardim Botânico do Rio de Janeiro. <http://floradobrasil.jbrj.gov.br/2010/FB023084>

Ferraz, R. C., Mello, A. A., Ferreira, R. A., \& Prata, A. P. N. (2013). Levantamento fitossociológico em área de caatinga no monumento natural Grota do Angico, Sergipe, Brasil. Revista Caatinga, 26(3), 89-98.

Fernandes, A. G. et al. Lonchocarpus araripensis Bentham. (1964). Boletim da Sociedade Cearence de Agronomia, 53(5), 184-189.

Fernandes, A. G., \& Bezerra, P. (1990). Estudo fitogeográfico do Brasil. Fortaleza: Stylus Comunicações.

Gardner, G. (1849). Travels in the interior of Brazil, principally through the northern Provinces and the Gold and Diamond Districts, during the years 18361841. London: Reeve, Bros.

Giulietti, A. M., Harley R. M., Queiroz, L. P. M., Barbos, A, R. V., Bocage Neta, A. L., \& Figueiredo, M. A. (2002). Plantas endêmicas da caatinga. In: Sampaio, E. V. S. B.,Giulietti,A. M., Virgínio,J., Gamarra-Rojas C. F. L. (Orgs.). Vegetação e flora das caatingas. Recife, PE: APNE / CNIP.

Govaerts, R., Frodin, D.G., \& Radcliffe-Smith, A. (2000). World Checklist and Bibliography of Euphorbiaceae and Pandaceae. The Royal Botanic Gardens, Kew, 2, 417-536.

Hunziker, A. T. (2001). The Genera of Solanaceae. Ruggell: A. R. G. Gantner.

IBGE. (2016). Instituto Brasileiro de Geografia e Estatística. <http://www.cidades.ibge.gov.br/.>

Knapp, S. A. (2008). Revision of the Solanumhavanensespecies group and New taxonomic additions to the Geminata Clade (Solanum, Solanaceae). Annalsofthe Missouri Botanical Garden, 95, 405-458.

Leitão, A. C., Vasconcelos, W. A., Cavalcante, A. M. B., Tinôco, L. B. M., \& Fraga, V. S. (2014). Florística e Estrutura de um Ambiente Transicional Caatinga-Mata Atlântica. Revista Caatinga, 27(3), 200-210.

Lima, I. B., \& Barbosa, M. R. V. (2014). Composição Florística da RPPN Fazenda Almas, no Cariri Paraibano, Paraíba, Brasil. Revista Nordestina de Biologia, 1, 49-67.

Mendonça, L. A. R., Frischkorn, H., Santiago, M. M. F., \& Filho, J. M. (2000). Qualidade da Água na Chapada do Araripe e sua Vulnerabilidade, 1st Joint World Congresson Groundwater, 1-16.

Moro, M. F., Lughadha, E. N., Filer, D. L., Araujo, F. S., \& Martins, F. R. (2014). A catalogue of the vascular plants of the Caatinga Phytogeographical Domain: a synthesis of floristic and phytosociological surveys. Phytotaxa, 160, 1- 118, 2014.

Nascimento, R. R. G., Pimenta, A. T. A., Neto, P. L., Junior, J. R. C., Costa-Lotufo, L. V., Ferreira, E. G., Tinoco, L. W., Braz-Filho, R., Silveira, E. R., \& Lima, M. A. S. (2015). New Alkaloids from Margaritopsis carrascoana (Rubiaceae). Journal of the Brazilian Chemical Society, 26(6), $1152-1159$.

Nee, M. (1999). Synopsis of Solanum in the world. In: M. Nee, D. E. Symon, R. N. Lester, J. P. Jessop (Orgs.). Solanaceae IV: Advances in Biology \& Utilization. Kew: Royal Botanic Gardens.

Neto, J. L. S. B. (2014). O gênero Sida L. (Malvaceae) no Estado de Pernambuco, Brasil (Dissertação de Mestrado). Universidade Federal Rural de Pernambuco - Programa de Pós-Graduação em Botânica, Recife, PE, Brasil.

Pereira Júnior, L. R., Andrade, A. P., \& Araújo, K. D. (2013). Composição Florística e Fitossociológica de um Fragmento de Caatinga em Monteiro, PB. HOLOS, 6(28), 73-87.

Queiroz, L. P. (2009). Leguminosas da Caatinga. Feira de Santana: Universidade estadual de Feira de Santana.

Radambrasil. (1981). Levantamento de Recursos Naturais. Folhas S.B., 23, 24-25. 
Research, Society and Development, v. 10, n. 13, e506101321398, 2021

Ribeiro-Silva, S., Medeiros, M. B., Gomes, B. M., Seixas, E. N. C., \& Silva, M. A. P. (2012). Angiosperms from the Araripe National Forest, Ceará, Brazil. CheckList, 8(4), 744-751.

Rodal, M. J. N., Costa, K. C. C., \& Silva, A. C. B. L. (2008). Estrutura da vegetação caducifólia espinhosa (Caatinga) de uma área do sertão central de Pernambuco. Hoehnea, 35(2), 209-217.

Sampaio, E. V. S., Andrade-Lima, D., \& Gomes, M. A. F. (1981). O Gradiente Vegetacional das Caatingas e Áreas Anexas. Revista Brasileira de Botânica, $4(1), 27-30$

Sanquetta, M. N. I., Corte, A. P. D., Sanquetta, C. R., Rodrigues, A. L., \& Mongon, F. (2014). Diversidade e Estrutura Fitossociológica da Caatinga na Regiãode Brumado - BA. Enciclopédia Biosfera, 10(17), 1-2157.

Santos, J. C., Leal, I. R., Almeida-Cortez, J. S., Fernandes, G. W., \& Tabarelli, M. (2009). Caatinga: The scientific negligence experienced by a dry tropical forest. Tropical Conservation Science, 4, 276-286.

Santos, W. S., Henriques, I. G. N., Santos, W. S., Ramos, G. G., Vasconcelos, G. S., \& Vasconcelos, A. D. M. (2017). Análise florística-fitossociológica e potencial madeireiro em área de caatinga submetida a manejo florestal. ACSA, 13(3), 203-211.

Silva, J. W. S., Dantas, I. C., Chaves, T. P., \& Felismino, D. C. (2010). Estudo Florístico do Sítio Arqueológico Pedra-do-Touro no município de Queimadas, PB. Revista de Biologia e Fármacia - BIOFAR, 4(2), 47-57.

Simon, M. F., Grether, R., de Queiroz, L. P., Särkinen, T. E., Dutra, V. F., \& Hughes, C. E. (2011). The evolutionary history of Mimosa (Leguminosae): toward a phylogeny of the sensitive plants. American Journal of Botany, 98(7), 1201-1221.

Siqueira Filho, J. A., Santos, A. P. B., Nascimento, M. F. S., \& Santo, F. S. E. (2009). Guia de Campo de Árvores da Caatinga. Petrolina: Editora e Gráfica Franciscana Ltda.

Tavares, F. M., Schulz, K., Pereira, R. C. A., Cierjacks, A., \& Almeida -Cortez, J. S. (2016). Floristic survey of the Caatinga in areas with different grazing intensities, Pernambuco, Northeast Brazil. Journalof Environmental AnalysisandProgress, 1(1), 43 - 51.

Viana, M. S., \& Neumann, V. H. L. (2006). O Membro Crato da Formação Santana, CE. In: Schobbenhaus, C., Campos, D. A., Queiroz, E. T., Winge, M., Berbert-Born, M. (Orgs.). Sítios Geológicos e Paleontológicos do Brasil. 